\title{
The formation of the coronal flow/ADAF
}

\author{
E. Meyer-Hofmeister and F. Meyer \\ Max-Planck-Institut für Astrophysik, Karl- Schwarzschildstr. 1, 85740 Garching, Germany \\ Received 23 December 2002 / Accepted 21 February 2003

\begin{abstract}
We develop a new method to describe the accretion flow in the corona above a thin disk around a black hole in vertical and radial extent. The model is based on the same physics as the earlier one-zone model, but now modified including inflow and outflow of mass, energy and angular momentum from and towards neighboring zones. We determine the radially extended coronal flow for different mass flow rates in the cool disk resulting in the truncation of the thin disk at different distance from the black hole. Our computations show how the accretion flow gradually changes to a pure vertically extended coronal or advection-dominated accretion flow (ADAF). Different regimes of solutions are discussed. For some cases wind loss causes an essential reduction of the mass flow.
\end{abstract}

Key words. accretion, accretion disks - black hole physics - X-rays: stars - galaxies: nuclei

\section{Introduction}

The new fascinating results from XMM-Newton and the Chandra X-ray Observatory allow a deeper insight into the physical processes in many astrophysical objects, on scales ranging from binary stars to galaxies. One of these topics is the accretion onto black holes. This can happen in form of an advection-dominated accretion flow (ADAF) or via a standard geometrically thin Shakura-Sunyaev accretion disk. Since an ADAF is only possible in the inner region around the central accretor one has in many objects an advection-dominated accretion flow in the inner region, fed by the mass flow through a standard disk at larger distances from the black hole (for a recent review on ADAF models see Narayan 2002). The situation is the same for accretion onto black holes in galactic X-ray binaries and onto supermassive black holes in active galactic nuclei (AGN).

The spectra arising from these two different ways of accretion are very different, a soft multi-temperature spectrum from the black-body like accretion disk and a hard power law spectrum from the Comptonizing much hotter gas in an ADAF (one observes the combination of both originating at different distances from the black hole). The observed change of the spectral type, the soft/hard spectral transitions in X-ray binaries, were successfully modeled as related to a changing mass flow rate by Esin et al. (1997, 1998), thereby supporting this picture of an ADAF in the inner region surrounded by a standard disk. This means that at a certain distance from the black hole the accretion mode changes from the flow via a thin disk to a hot

Send offprint requests to: E. Meyer-Hofmeister, e-mail: emm@mpa-garching.mpg.de gas flow. The distance where this happens depends on the mass flow rate from outside.

Meyer et al. (2000) developed a model for a corona above a geometrically thin standard disk around a black hole (basically the same physics as already discussed earlier for disks of dwarf nova systems, where the compact object is a white dwarf (Meyer \& Meyer-Hofmeister 1994)). The corona is fed by gas which evaporates from the cool thin disk underneath. An equilibrium establishes between the cool accretion stream and the hot flow. The efficiency of this process increases towards the black hole. This means that at a certain distance all matter is evaporated and the disk is truncated. From this distance on inward all gas is in the hot flow and proceeds towards the black hole as an advection-dominated flow. This original model is a simplifying description of the two-dimensional accretion flow (of vertical and radial extent with a free boundary condition on a radially extended surface). The resulting evaporation efficiency as a function of distance (and of the central black hole mass) allows to determine the location at which the thin disk is truncated.

For some X-ray binaries in quiescence the location of the inner edge of the disk can be deduced from the orbital velocity there, which is inferred from the observed $\mathrm{H}_{\alpha}$ line profiles. This requires that the disk temperature at the innere edge does not exceed the ionization temperature of hydrogen. Using the ADAF model for the fit to the spectra of soft X-ray transients as e.g. A620-00, V404 Cyg and Nova Mus 1991 the mass accretion rates were derived (Narayan et al. 1996, 1997). If one compares these results with the disk truncation radius from the evaporation model the agreement is reasonable (Liu et al. 1999; Meyer-Hofmeister \& Meyer 1999). The change from disk accretion to an ADAF was also investigated for several low 
luminosity AGN and elliptical galaxies (Quataert et al. 1999; Gammie et al. 1999; Di Matteo et al. 1999, 2000), also the theoretically expected disk truncation (Liu \& Meyer-Hofmeister 2001). The results confirm that the truncation of the thin disk is located at smaller distance from the black hole for higher mass flow rates in the thin disk. Only for low luminosity AGN the observed spectra seem to demand a disk truncation at radii too small for the accretion rate (Quataert et al. 1999). But this apparent discrepancy might be resolved by the effect of magnetic fields from a dynamo in the underlying disk on the coronal gas flow (Meyer \& Meyer-Hofmeister 2002).

We now present work which is an essential step beyond the one-zone model. We develop a new method to describe the accretion flow in the corona above a thin disk in its vertical and radial extent. The model is based on the same physics as in the one-zone model, but now modified including inflow and outflow of mass, energy and angular momentum from and towards neighboring zones. In the earlier model inflow from outward regions was neglected. But this is necessary if one considers regions inside the evaporation maximum (in the one-zone model at about 300 Schwarzschild radii, compare Fig. 3 in Meyer et al. 2000). For the inner regions it is also important to take into account different ion and electron temperature and, in the case of a high mass flow rate in the thin disk, the effect of Compton cooling of coronal electrons by disk photons. Both were included in the one-zone model description by Liu et al. (2002), where emphasis was put on evaluation of the coronal flow in the case of high accretion rates as in narrow-line Seyfert 1 galaxies.

The co-existence of hot and cold gas around galactic black holes and in AGN was also investigated by Różańska \& Czerny (2000a,b). Different ion and electron temperatures were already included. In their work the physical picture is basically the same as in ours, but the results differ in detail. The investigation focuses on the innermost region near the black hole. In earlier work (Meyer-Hofmeister \& Meyer 2001) we discussed the difference of the results. Processes of evaporation very close to the black hole were studied by Spruit \& Deufel (2001).

In Sect. 2 we discuss the physics of interaction between the hot corona and a cool disk underneath. We introduce the modifications necessary for a consistent treatment of the mass, energy and angular momentum flow. We describe the procedure how one finds the solution for the two-dimensional accretion flow, put together from the separately computed structures for different radii, and we present the new computational results (Sect. 3). This multi-zone model describes the increase of the mass flow in the hot corona towards the black hole. A part of the matter is lost in a wind from the hot corona. In Sect. 4 we discuss the different regimes of solutions, wind loss from the coronal flow and the consequences of a varying mass flow rate in the thin disk on spectral transitions.

\section{The physics of the interaction of the hot corona and a cool disk underneath}

If we imagine hot gas above cool matter in a disk below both layers interact. This interaction happens in the gravitational potential of the central star (as earlier considered near the white dwarf in binaries, a "siphon flow", Meyer \& Meyer-Hofmeister 1994). The hot corona conducts heat downward by electron conduction. At the bottom the temperature decreases from its high coronal value to a low chromospheric value, heat conduction becomes ineffective and the thermal heat flow has to be radiated away. The efficiency of radiation (in the optical thin case) depends on the square of the particle number density. If this density is too small the material will heat up and increase the density in the corona. In this way the corona of a given temperature will "dig" itself so deep into the chromospheric layers that a density is reached which is able to radiate away the downward heat conduction and an equilibrium establishes between the cool accretion stream and the hot flow. The final density in the corona is determined by the pressure equilibrium at the interface. The hot flow continuously drains mass from the corona towards the central object. This is resupplied by evaporation from the surface of the cool disk as the corona tries to restore the density to the stationary level.

\subsection{The modeling of the corona above the thin disk in vertical and radial extent}

To describe the structure of the corona above the cool disk we take the standard equations of viscous hydrodynamics: conservation of mass, the equations of motion and the first law of thermodynamics. We want to determine the vertical structure of the corona at different distances $r$ from the black hole with emphasis on the inner accretion regions. For the earlier "one-zone model" only the zone at the edge of the thin disk was considered. Since the evaporation efficiency increases steeply in radial direction inward this approach gives already good results (but only for distances from the black hole where the evaporation efficiency indeed increases inward). We now want to investigate the coronal structure in its full extension in two dimensions. We determine the vertically extended coronal structure for a series of successive radial zones. In this multi-zone model the divergence in radial direction is replaced by inflow/outflow of mass and angular momentum in the zones (in the former one-zone model this could only be taken into account approximately).

\subsection{The equations}

We use cylindrical coordinates $r, \varphi, z$ with the $z$-axis perpendicular to the disk midplane. We consider stationary and azimuthally symmetric flows. We use basically the same equations as for the one-zone model, also used in Liu et al. (2002), but modified to take mass, energy and angular momentum inflow and outflow in its radial dependence into account.

In the following we list these five ordinary differential equations. The dependent variables are the vertical mass flow $\dot{m}=\rho v_{z}$ ( $\rho$ density, $v_{z}$ vertical flow velocity), the vertical component of the heat flux $F_{\mathrm{c}}$, the pressure $P$ and the ion and electron temperature $T_{\mathrm{i}}$ and $T_{\mathrm{e}}$, the independent variables are $r$ and $z$.

We use the following equation of state

$P=n_{\mathrm{i}} k T_{\mathrm{i}}+n_{\mathrm{e}} k T_{\mathrm{e}} \approx \frac{\mathfrak{R} \rho}{2 \mu_{0}}\left(T_{\mathrm{i}}+T_{\mathrm{e}}\right)$, 
with $n_{\mathrm{i}}, n_{\mathrm{e}}, T_{\mathrm{i}}, T_{\mathrm{e}}$ ion and electron number density and respectively temperature. $\rho_{\mathrm{i}}=\rho$ is the ion mass density. We take a standard chemical composition $(X=0.75, Y=0.25)$ in the corona and the average molecular weight then $\mu_{0}=0.62$. In a fully ionized gas of standard composition the electron number density is about $10 \%$ larger than the ion number density. The last expression for the total pressure in Eq. (1) is chosen for simplicity. For $\mu_{0}=0.62$ it is exact if $T_{\mathrm{e}}=T_{\mathrm{i}}$, but it overestimates $P$ for $T_{\mathrm{e}} \ll T_{\mathrm{i}}$ by $5 \%$. We neglect this difference.

From the conservation of mass we get our first equation

$\frac{\mathrm{d}}{\mathrm{d} z}\left(\rho v_{z}\right)=\eta_{\mathrm{M}} \cdot \frac{2}{r} \rho v_{\mathrm{r}}-\frac{2 z}{r^{2}+z^{2}} \rho v_{z}$,

with $v_{\mathrm{r}}$ the radial diffusive velocity and $\eta_{\mathrm{M}}$ the mass advection modification term (see next section). The second term in Eq. (2) approximately takes into account the effect of the changing channel cross section $\approx\left(1+z^{2} / r^{2}\right)$, for the ascending flow as its shape changes from cylindrical to spherical at large height $z$ (compare Meyer et al. 2000).

The second equation is the $z$-component of the equation of motion,

$\rho v_{z} \frac{\mathrm{d} v_{z}}{\mathrm{~d} z}=-\frac{\mathrm{d} P}{\mathrm{~d} z}-\rho \frac{G M z}{\left(r^{2}+z^{2}\right)^{3 / 2}}$,

with $G$ gravitational constant and $M$ mass of the central black hole. The $r$-component of the equation of motion to first order gives the rotational velocity $v_{\varphi}$ as equal to the local Kepler velocity, $v_{\varphi}^{2}=G M r^{2} /\left(r^{2}+z^{2}\right)^{3 / 2}$, neglecting radial pressure gradients, small of order $\mathfrak{R T r} / G M \mu \leq \frac{1}{5}$ in our solutions. $T$ stands for the temperature which determines the hydrostatic layering, $T \approx T_{\mathrm{i}}$. The $\varphi$-component yields angular momentum conservation and determines $v_{\mathrm{r}}$.

The vertical conductive heat flux provides our third equation

$F_{\mathrm{c}}=-\kappa_{0} T_{\mathrm{e}}^{5 / 2} \frac{\mathrm{d} T_{\mathrm{e}}}{\mathrm{d} z}$

with $\kappa_{0}=10^{-6} \mathrm{~g} \mathrm{~cm} \mathrm{~s}^{-3} \mathrm{~K}^{-7 / 2}$ for a fully ionized plasma (Spitzer 1962). This formula for the heat conduction is valid if the electron mean free path is small compared to the length over which the temperature changes significantly. In the region of our solution where heat conduction is a significant contribution this is the case (occasionally at least marginally).

The two remaining equations, the energy equations for ions and for electrons, as derived by Liu et al. (2002), include the cooling and heating processes in the hot corona and the equations are now modified for the multi-zone model. For ions the energy balance is determined by viscous heating, cooling by collision with electrons and radial and vertical advection. The friction is taken proportional to the pressure with a standard $\alpha$-prescription.

$\frac{\mathrm{d}}{\mathrm{d} z}\left(\rho_{\mathrm{i}} v_{z} u_{\mathrm{i}}\right)=\frac{3}{2} \alpha P \Omega-q_{\text {ie }}+\eta_{\mathrm{E}} \cdot \frac{2}{r} \rho_{\mathrm{i}} v_{\mathrm{r}} u_{\mathrm{i}}-\frac{2 z}{r^{2}+z^{2}}\left(\rho_{\mathrm{i}} v_{z} u_{\mathrm{i}}\right)$,

with $\Omega$ the rotational frequency, $\rho_{\mathrm{i}}$ ion density, $\alpha$ viscosity parameter. $u_{\mathrm{i}}$ is the specific energy of ions

$u_{\mathrm{i}}=\left(\frac{v^{2}}{2}+\frac{\gamma}{\gamma-1} \frac{P_{\mathrm{i}}}{\rho_{\mathrm{i}}}+\Phi\right)$, with $\Phi$ the (Newtonian) potential

$\Phi=-\frac{G M}{\left(r^{2}+z^{2}\right)^{1 / 2}}$.

$\frac{3}{2} \alpha P \Omega$ is the viscous heating rate per unit volume and $q_{\text {ie }}$ the rate of energy transfer from ions to electrons (Stepney 1983) as taken in Liu et al. (2002). The energy advection modification term $\eta_{\mathrm{E}}$ is discussed in the next section. The contribution of the frictional stress to the divergence of the energy flow is already explicitly taken into account as frictional dissipation term in the equations.

For the multi-zone model there are two changes compared to the one-zone model, (1) the mass and energy modification factors and (2) we now take into account the radial dependence of $v_{\varphi}^{2}$ in the formula for the specific energy (see Sect. 2.3.3).

For electrons the energy balance is determined by the processes of heating by collisions with ions, cooling by bremsstrahlung (free-bound and free-free transitions), Compton cooling, and vertical thermal conduction. We neglect here the radial thermal conduction in a first approximation. In the main part of the vertical structure solution it is not a dominant term (see Meyer et al. 2000).

The second energy equation is

$$
\begin{aligned}
\frac{\mathrm{d}}{\mathrm{d} z}\left(\rho_{\mathrm{e}} v_{z} u_{\mathrm{e}}+F_{\mathrm{c}}\right)= & q_{\mathrm{ie}}-n_{\mathrm{e}} n_{\mathrm{i}} L\left(T_{\mathrm{e}}\right)-q_{\mathrm{comp}}+\eta_{\mathrm{E}} \cdot \frac{2}{r} \rho_{\mathrm{e}} v_{\mathrm{r}} u_{\mathrm{e}} \\
& -\frac{2 z}{r^{2}+z^{2}}\left(\rho_{\mathrm{e}} v_{z} u_{\mathrm{e}}+F_{\mathrm{c}}\right),
\end{aligned}
$$

where $u_{\mathrm{e}}$ is specific energy of electrons (defined correspondingly to $\left.u_{\mathrm{i}}\right)$ and $n_{\mathrm{e}} n_{\mathrm{i}} L\left(T_{\mathrm{e}}\right)$ is the bremsstrahlung cooling rate and $q_{\text {comp }}$ the Compton cooling rate (Rybicki \& Lightman 1979), as in Liu et al. (2002).

In the energy balance for the electrons one could omit the terms multiplied with $\rho_{\mathrm{e}}$ due to the low ratio of electron to ion mass. We keep the equation for ions, Eq. (5), and replace the equation for electrons by a joint equation for electrons and ions (with $u$ the specific energy of electrons and ions).

\subsection{The modification terms for radial inflow/outflow}

We first derive the formula for the radial diffusive velocity and then the mass and energy advection modification terms. For simplicity these modifications are taken in a height averaged way.

\subsubsection{The radial diffusive velocity}

The equation for local angular momentum conservation is

$\frac{\partial}{\partial r}\left(2 \pi r \rho v_{\mathrm{r}} r^{2} \Omega\right)+\frac{\partial}{\partial z}\left(2 \pi r \rho v_{z} r^{2} \Omega\right)+\frac{\partial}{\partial r}\left(2 \pi r \tau_{\mathrm{r} \varphi}\right)=0$

with the frictional stress $\tau_{\mathrm{r} \varphi}$

$\tau_{\mathrm{r} \varphi}=-\alpha P=\mu r \frac{\partial \Omega}{\partial r} \approx-\frac{3}{2} \Omega \mu$,

$\mu$ viscosity. For the lower, dominant part of the corona it is a reasonable approximation to neglect the $z$-dependence of $\Omega$ (for an error estimate see Meyer et al. 2000). Mass conservation

$\frac{\partial}{\partial r}\left(2 \pi r \rho v_{\mathrm{r}}\right)+\frac{\partial}{\partial z}\left(2 \pi r \rho v_{z}\right)=0$ 
together with $\frac{\partial}{\partial z}\left(r^{2} \Omega\right) \approx 0$ yields

$2 \pi r \rho v_{\mathrm{r}} \frac{\partial}{\partial z}\left(r^{2} \Omega\right)+3 \pi \frac{\partial}{\partial r}\left(r^{2} \Omega \mu\right)=0$.

Using

$\frac{\partial\left(r^{2} \Omega\right)}{\partial r} \approx \frac{1}{2} \frac{r^{2} \Omega}{r}$

one finally obtains for the radial diffusive velocity (inward directed)

$v_{\mathrm{r}}=-\frac{3 \alpha P}{r \Omega \rho}\left(1+2 \frac{\partial \ln \mu}{\partial \ln r}\right)=-\frac{\alpha V_{\mathrm{s}}^{2}}{v_{\mathrm{K}}}\left(1+2 \frac{\partial \ln \mu}{\partial \ln r}\right)$,

with $v_{\mathrm{K}}$ Kepler velocity. As a simplification we take the rate of change of the viscosity the same at all heights $z$ and replace the logarithmic derivative of $\mu$ by the logarithmic derivative of its integral $f$

$\frac{\partial \ln \mu}{\partial \ln r}=\frac{\mathrm{d} \ln f}{\mathrm{~d} \ln r}, \quad f=2 \int_{z_{0}}^{z_{1}} \mu \mathrm{d} z$

(integration over the height of the corona on both sides of the disk). $f$ results from the computed vertical distribution of the pressure according to Eq. (10). With this replacement Eqs. (10), (14) and (15) yield for the coronal accretion rate (positive if inward)

$$
\begin{aligned}
\dot{M}_{\mathrm{c}} & =2 \int_{z_{0}}^{z_{1}} 2 \pi r \rho v_{\mathrm{r}} \mathrm{d} z=3 \pi f\left(1+2 \frac{\mathrm{d} \ln f}{\mathrm{~d} \ln r}\right) \\
& =-2 \pi r \Sigma \bar{v}_{\mathrm{r}}
\end{aligned}
$$

the third equality defining the mean radial velocity $\bar{v}_{\mathrm{r}}$, with the surface density $\Sigma=2 \int_{z_{0}}^{z_{1}} \rho \mathrm{d} z$ (both sides of disk).

\subsubsection{The divergence of mass and energy flow}

We derive the modifications of the sidewise advection terms in the equations for conservation of mass and energy again as mean values over $z$. The radial divergence is written as

$$
\frac{1}{r} \frac{\mathrm{d}}{\mathrm{d} r}\left(r \sum \bar{v}_{\mathrm{r}}\right)=-\frac{2}{r} \Sigma \bar{v}_{\mathrm{r}} \eta_{\mathrm{M}}
$$

such that $\eta_{\mathrm{M}}$ measures the radial divergence in terms of its onezone model value. Thus follows

$\eta_{\mathrm{M}}=-\frac{1}{2} \frac{\mathrm{d} \ln \left|r \sum \bar{v}_{\mathrm{r}}\right|}{\mathrm{d} \ln r}=-\frac{1}{2} \frac{\mathrm{d} \ln \left|\dot{M}_{\mathrm{c}}\right|}{\mathrm{d} \ln r}$.

With Eq. (16) the mass modification factor can also be written in the form

$\eta_{\mathrm{M}}=-\frac{1}{2} \frac{\mathrm{d} \ln \left|f\left(1+2 \frac{\mathrm{d} \ln f}{\mathrm{~d} \ln r}\right)\right|}{\mathrm{d} \ln r}$.

The divergence of the energy advection involves the derivative of $\dot{M}_{\mathrm{c}} \cdot u(r, \eta)$ with $u$ the specific energy (see below),

$\eta_{\mathrm{E}}=-\frac{1}{2} \frac{\mathrm{d} \ln \left|\dot{M}_{\mathrm{c}}(r, \eta) \cdot u(r, \eta)\right|}{\mathrm{d} \ln r}$.

The potential, kinetic, and thermal specific energies all scale approximately as $1 / r$ (see Liu et al. 2002, Fig. 4). The factor then is

$\eta_{\mathrm{E}}=\eta_{\mathrm{M}}+0.5$

\subsubsection{The specific energy}

In the specific energy $u$ the kinetic energy is $\frac{v^{2}}{2}=\left(v_{\mathrm{r}}^{2}+v_{z}^{2}+\right.$ $\left.v_{\varphi}^{2}\right) / 2$. Since $v_{\varphi}$, the dominant term, is $r$ dependent, and it has to be taken into account as $v_{\varphi}^{2}=G M r^{2} /\left(r^{2}+z^{2}\right)^{3 / 2}$ (in the onezone model it was taken as constant and dropped out of the equations).

\subsection{Boundary conditions}

The boundary conditions are the same as those in the one-zone model.

As the lower boundary of our calculations at $z=z_{0}$, we take the level where electron temperature and ion temperature are already about the same and have the value $T_{\mathrm{e}}=T_{\mathrm{i}}=10^{6.5} \mathrm{~K}$. Below an analytic solution suffices to cover the small remaining extent before the disk chromosphere is reaching it and yields a relation between pressure and downward heat flux (for a more detailed discussion see Meyer et al. 2000).

At the upper boundary with no pressure confinement at infinity we require sound transition at some height $z=z_{1}$ (free boundary), $v_{z}=V_{\mathrm{s}}$. Further with no influx of heat from infinity there, we require $F_{\mathrm{c}}=0$ neglecting a small remaining outward heat flow.

This constitutes 5 boundary conditions for the 5 ordinary differential equations in $z$.

\section{A consistent solution at all distances $r$}

The equations described are valid for accretion in different astrophysical objects, stellar and galactic black hole masses. The solutions scale with Eddington accretion rate and Schwarzschild radius. In the following we present the solutions for an accretion flow around a $6 M_{\odot}$ black hole (the Eddington accretion rate then is $8.4 \times 10^{18} \mathrm{~g} / \mathrm{s}$, the Schwarzschild radius $1.8 \times 10^{6} \mathrm{~cm}$ ). We take the viscosity parameter $\alpha=0.3$ (for the dependence of the coronal structure on the viscosity see Meyer-Hofmeister \& Meyer 2001).

\subsection{How to find consistent solutions}

The main goal of the new approach is to find out how the corona is stretched out above the disk in radial extent. The one-zone model gives a description of the coronal structure only for the one inner zone where the thin disk underneath becomes truncated. This is a reasonable first approach when the evaporation efficiency is steeply increasing with decreasing $r$ and evaporation is the dominant mass feeding cause there. (Mass flow into the corona from farther outward was neglected and simple estimates for the flow of mass, energy and angular momentum through this one zone were made.)

For the determination of the coronal mass flow in a radially extended region the difference of inflow and outflow in a succession of radial zones is now properly accounted for. This means that the coronal mass flow at a certain radius depends on the coronal structure in the neighboring zones and must be determined iteratively. 


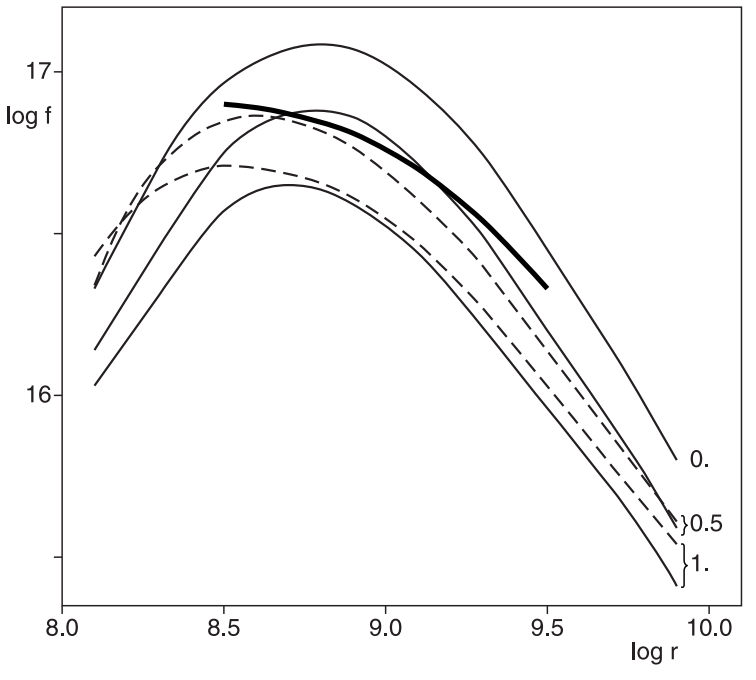

Fig. 1. Values of the viscosity integral $f\left(r, \tilde{\eta}_{\mathrm{M}}, \tilde{\eta}_{\mathrm{E}}\right)$ for different parameters, basic curves for the evaluation of the coronal flow. solid lines: $\left(\tilde{\eta}_{\mathrm{M}}, \tilde{\eta}_{\mathrm{E}}\right)=(0,0),(0.5,0.5),(1,1)$; long dashes: $\left(\tilde{\eta}_{\mathrm{M}}, \tilde{\eta}_{\mathrm{E}}\right)=(0.5,1)$, $(1,1.5)$ For $(0,0.2)$ the line is approximately the same as for $(0,0)$. Thick line: example of a consistent curve $\log f$, including the mass, energy and angular momentum flow consistently (in our computations we investigate the accretion flow around a $6 M_{\odot}$ black hole).

We first compute the coronal structure for successive distances and a series of values for the modification terms to construct a grid of curves $f\left(r, \eta_{\mathrm{M}}, \eta_{\mathrm{E}}\right)$. The derivative of $f$ only appears in $v_{\mathrm{r}}$ and in the modification terms. We therefore take these terms together as

$\tilde{\eta}_{\mathrm{M}}=\eta_{\mathrm{M}} \cdot\left(1+2 \cdot \frac{\mathrm{d} \ln f(r, \eta)}{\mathrm{d} \ln r}\right)$
$\tilde{\eta}_{\mathrm{E}}=\eta_{\mathrm{E}} \cdot\left(1+2 \cdot \frac{\mathrm{d} \ln f(r, \eta)}{\mathrm{d} \ln r}\right)$.

We show some of these results in Fig. 1. A value $\tilde{\eta}_{M}=0$ means a net zero outflow. In this case we found that the structure approximately is independent of the value of $\tilde{\eta}_{\mathrm{E}}$. The value of the viscosity integral $f$ decreases with increasing net flow. Higher values of $f$ correspond to higher values of the pressure at the bottom of the corona. The value $\tilde{\eta}_{\mathrm{M}}=1$ corresponds to the net outflow taken in the one-zone model (aside from the now included term $v_{\varphi}^{2}$ ). From Fig. 1 we see that advection of energy has a different effect on the coronal structure at different distances $r$. This depends on whether the frictional energy release preferentially goes into radiation or into advective loss (compare Fig. 5 Meyer et al. 2000).

What we finally need is a series of coronal structures at each distance determined for given $\tilde{\eta}$ values $(\tilde{\eta}$ indicates the dependence on both $\tilde{\eta}_{\mathrm{M}}$ and $\tilde{\eta}_{\mathrm{E}}$ ) so that the curve $\log f(r, \tilde{\eta})$ has the slope $s=\mathrm{d} \ln f / \mathrm{d} \ln r$ that fits to these values taken for $\tilde{\eta}$. From Eqs. (19), (21), and (22) and the definition of $s$ we obtain

$\tilde{\eta}_{\mathrm{M}}=-\frac{1}{2}\left(s+s^{2}+2 \frac{\mathrm{d} s}{\mathrm{~d} \ln r}\right), \quad \tilde{\eta}_{\mathrm{E}}=\left(\tilde{\eta}_{\mathrm{M}}+0.5\right)(1+2 s)$.

This implies an ordinary differential equation of second order for $f(r)$. A unique solution then requires two boundary conditions, e.g. at an inner and an outer edge. The solutions thus form a two-dimensional manifold. Different approaches might be possible. An iterative procedure without enforcing appropriate boundary conditions, starting from one of our curves in Fig. 1, determining the derivatives of $\log f$ and the $\tilde{\eta}$ values and then the appropriate new $\log f$ curves, seems not to converge to a consistent solution. This is probably due to the large number of possible solutions corresponding to the diversity of possible boundary conditions.

We use the following procedure. We start at a chosen distance $r=r_{\text {tr }}$ where the thin disk is truncated. There the boundary condition is $s\left(r_{\text {tr }}\right)=0$ which means that all angular momentum carried inward into the inner disk free region is returned by friction (see discussion in Meyer et al. 2000). Now we construct a consistent $\log f$ curve step after step. Step 1: we choose an initial value of $\log f(r, \tilde{\eta})$ (from our computed grid of curves); from Eq. (23) we see which value has to be chosen for $\frac{\mathrm{d} s}{\mathrm{~d} \ln r}$ to give the values $\tilde{\eta}_{\mathrm{M}}$ and $\tilde{\eta}_{\mathrm{E}}$ that belong to the chosen initial value of $f$ (the derivative is approximated by a difference quotient $\left(s\left(\log r_{\text {tr }}+\Delta \log r\right)-s\left(\log r_{\text {tr }}\right) / \Delta \log r\right.$, we used $\Delta \log r=0.2$ ). This determines the slope $s$ with which to proceed to the next $\log f$ value at the next radius. Step 2: the slope determined in step 1 yields the next value for $\log f$; again we determine the further slope so that relation (23) is fulfilled. Each step thus determines the further outward slope $s$ of the $\log f$ curve so that the $\tilde{\eta}$ values and $f(r, \tilde{\eta})$ are consistent.

For a given truncation radius and for each initial value of $\log f$ we get with this procedure one consistent $\log f(r, \tilde{\eta})$ curve. A second boundary condition at an outer boundary of the interval for which we determine the coronal flow allows to discriminate between the various curves that belong to one truncation radius. The slope at the outer distance is a possible boundary condition. For $s=1 / 2$ the radial diffusive velocity $v_{\mathrm{r}}$ becomes zero, no mass flow inward or outward. Then closer to the black hole the mass flows inward, at larger distance the mass flows outward. In different disks the outer boundary condition might be different. In the case of X-ray binaries it seems plausible that tidal forces at the disk boundary prevent outer coronal mass outflow, that is $\mathrm{d} \ln f / \mathrm{d} \ln r$ becomes $-1 / 2$ there.

\subsection{Computational results}

Our method yields the coronal structure solutions for given truncation radii. This gives the mass flow rate in the corona at the truncation radius where no mass flows in the cool disk anymore. This mass flow together with the wind loss from the corona added up over the radial extent of the corona is equal to the mass flow in the far out cool disk. This establishes the dependence of the disk truncation radius on the mass flow rate in the outer thin disk. In Fig. 1 we show one $\log f$ curve starting at $\log r_{\text {tr }}=8.5$. In Fig. 2 we show three characteristic curves starting at $\log r_{\text {tr }}=8.5$ as well as the characteristic run of $\log f$ curves for other truncation radii. Generally the lower the initial of $\log f$ the steeper is the corresponding $\log f$ curve. One also sees that a small variation in the initial value of $f$ yields a large variation of the radius where $v_{\mathrm{r}}=0$ (marked by a diamond in Fig. 2). This indicates a somewhat weak dependence of the inner coronal mass flow rate on the outer boundary condition. 


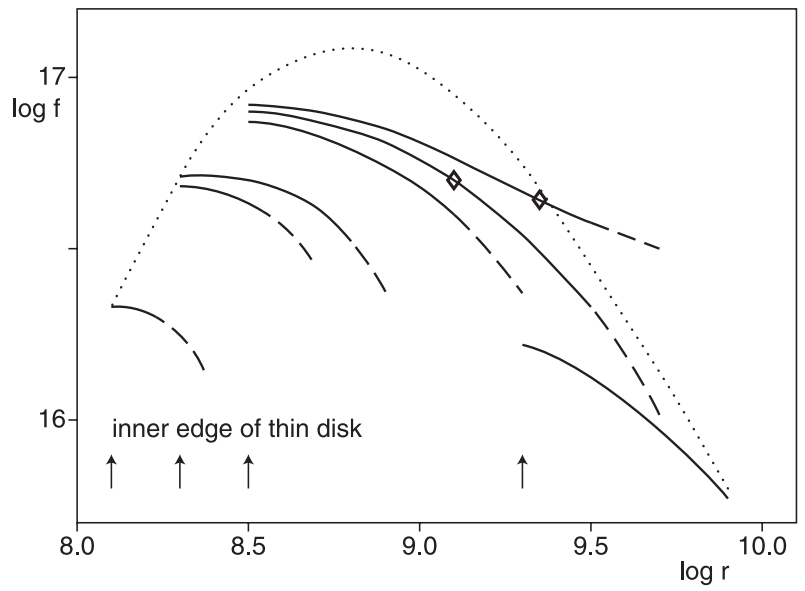

Fig. 2. Consistent solutions for $\log f$ for truncation radii $\log r=8.1$ to 9.3. Diamonds on two curves mark where $v_{\mathrm{r}}=0$. The coronal flow at the truncation radius is proportional to $f$. Dashed parts indicate uncertain extrapolation outside of the computed basic grid curves. The dotted curve is identical with the uppermost curve in Fig. 1 (accretion flow around a $6 M_{\odot}$ black hole).

Comparing the $f$ curves of the solutions with the field which our basic grid curves cover in the $\log f$-log $r$ diagram we see that the solution $f$ - curves can easily lead into areas outside of the grid, either to higher or lower values of $\log f$. If they stay within the range of computed basic curves necessarily the slope becomes less than $-1 / 2$. No consistent solutions have been constructed which have $v_{\mathrm{r}}=0$ at a very large distance. The too steeply decreasing curves correspond to the situation that the coronal flow is inward only near the inner boundary but turns into an outflow already a short distance away from it. To continue these curves correctly we need the coronal structures for high $\tilde{\eta}_{\mathrm{M}}$ values, which means a high net radial mass outflow. The flatter curves also can leave the field of standard solutions, that is $\tilde{\eta}_{\mathrm{M}} \leq 0$. The corona would then have a negative radial net outflow, which means less mass radially leaves the zone at the inner radius than enters into it at the outer radius.

In Fig. 3 we show the coronal mass flow for the case of a thin disk with a mass flow rate of $2.4 \times 10^{18} \mathrm{~g} / \mathrm{s}$ from outside. This disk becomes truncated at $\log r_{\text {tr }}=8.5$. We show how the coronal flow increases inward (together with the mass and energy flow modification values $\tilde{\eta}_{\mathrm{M}}$ and $\tilde{\eta}_{\mathrm{E}}$ of this consistent solution). The evaporation rate measured by $\dot{M}_{\text {evap }}=2 \pi r^{2} \dot{m}_{0}$ $\left(\dot{m}_{0}\right.$ vertical mass flow rate per unit surface at the bottom of the corona, in units of $\mathrm{g} / \mathrm{cm}^{2} \mathrm{~s}$ ) gives the amount of gas locally evaporated into the corona. The earlier one-zone model only considered evaporation in the innermost region. Here our solution shows a radially quite extended evaporation region. In the lower panel of Fig. 3 we show how the mass flow in the thin disk decreases inward. Gas evaporates into the hot coronal flow, from which a significant part, $\approx 50 \%$ is lost in a wind.

In Fig. 4 we show the results from a series of computations with successive truncation radii. We chose solutions where $v_{\mathrm{r}}=0$ occurs relatively far outward and give the coronal mass flow rates at the truncation radii. In addition we show the virial and ion and electron temperatures of the corona at height $z=r$ at the truncation radius. Note the small difference
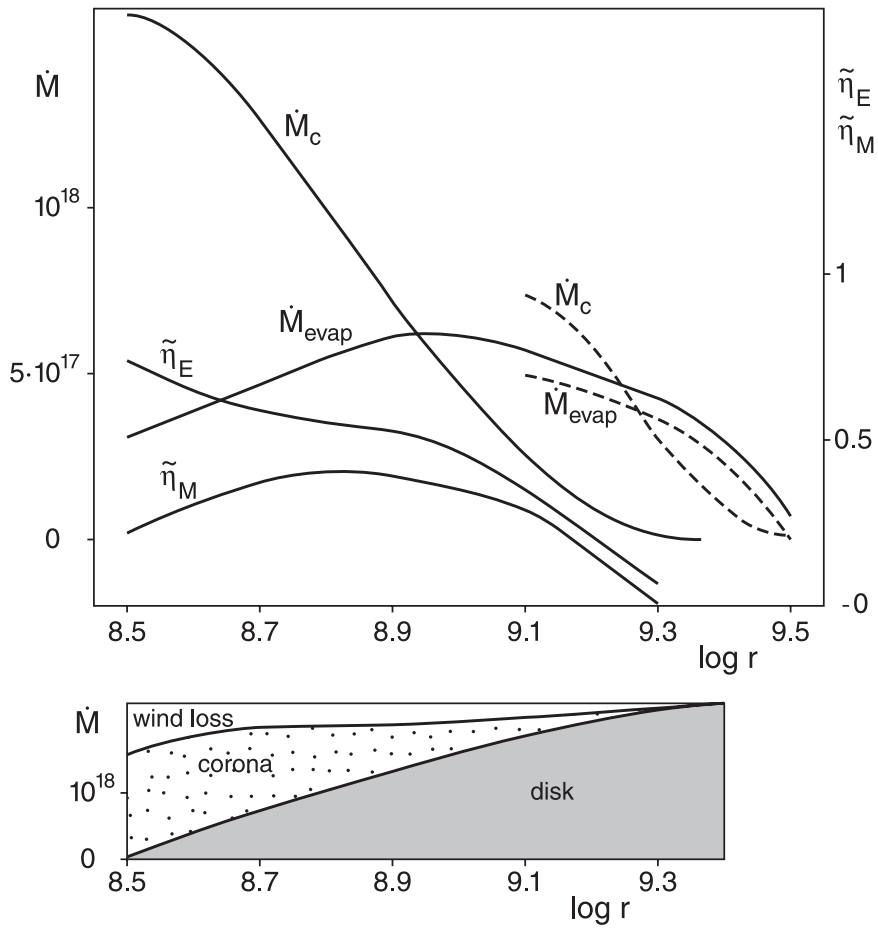

Fig. 3. Upper panel: solid lines: $\dot{M}_{\mathrm{c}}$ coronal mass flow rate above the thin disk around a $6 M_{\odot}$ black hole, truncated at $\log r_{\mathrm{tr}}=8.5$, mass and energy flow modification values $\tilde{\eta}_{\mathrm{M}}$ and $\tilde{\eta}_{\mathrm{E}} \cdot \dot{M}_{\text {evap }}$ evaporation rate (see text). Dashed lines: $\dot{M}_{\mathrm{c}}$ and $\dot{M}_{\text {evap }}$ for disk truncation at $\log r_{\text {tr }}=9.1$. Lower panel: mass flow rate in the thin disk (gray area) and in the corona (dotted area) as function of distance $r$ from the black hole, area remaining above indicates the rate of gas loss in the wind from the corona.

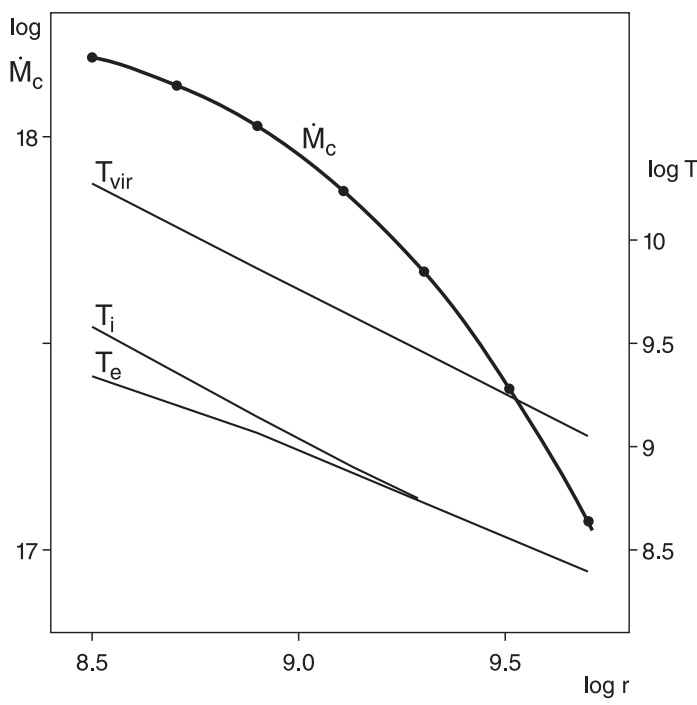

Fig. 4. Thick line: series of solutions for the coronal mass flow rate $\dot{M}_{\mathrm{c}}$, for disk truncations at $\log r$ from 8.5 to 9.7 ; thin lines: ion and electron temperature at coronal height $z=r$ together with the virial temperature $T=\frac{\mu}{\mathfrak{R}} \frac{G M}{r}$.

between $T_{\mathrm{i}}$ and $T_{\mathrm{e}}$. The coronal mass flow rates are higher than the ones derived with the one-zone model. This is in part due to inclusion of the value of $v_{\varphi}^{2}$ in the specific energy. 


\section{Discussion and conclusions}

\subsection{The nature of the solutions - wind loss}

Having developed the procedure for solving the differential equations described above we see that there are quite different regimes of solutions. Up to now we have studied solutions with a moderate advective mass and energy net outflow. These solutions seem adequate to describe the coronal flow above a thin accretion disk e.g. in X-ray binaries. For the investigation of AGN disks another outer boundary condition might be taken, but this would not affect the solutions essentially. It will be interesting also to study other regimes of solutions i.e. solutions leading into the area above our network of curves $\log f\left(r, \tilde{\eta}_{\mathrm{M}}, \tilde{\eta}_{\mathrm{E}}\right)$ in the $\log f$-log $r$ diagram. Test computations showed that in these solutions an increasing part of the coronal flow does not proceed inward but is lost in a wind. In our equations flaring vertical columns are considered. If the wind loss becomes important the structure becomes dependent on the assumed geometry. This geometry then has to be considered carefully to have a consistent picture how the expanding wind structure is arranged radially. This is an interesting topic in view of the question how much gas actually enters the disk free inner region and flows towards the black hole.

\subsection{Disk truncation closer to the black hole?}

If we imagine that the mass flow rate in the thin disk can be arbitrarily high we should encounter a situation where the disk is not yet truncated at e.g. $\log r=8.5$. If evaporation continues a truncation farther inward is possible. We have not yet found a consistent solution for the coronal flow corresponding to a disk truncation farther in. As can be seen from our solutions starting at larger distances we would have to start with higher initial values of $\log f$, values for which we have no $\log f$ curves. Logically, a coronal structure for condensation of gas instead of evaporation should then be appropriate. Such condensation already appears in the algebraic treatment of coronal structure by Różańska \& Czerny (2000a). Thus in our approach which consistently derives advective terms from the radial gradients condensation should also be expected. In the case of a net mass inflow by sideways advection part of the gas flows down into the cool disk while another part flows upward and leaves as a wind.

\subsection{Compton effect}

The computed coronal structure is valid for a mass flow rate in the thin disk low enough not to affect the corona by Compton cooling. Results in the work of Liu et al. (2001, Fig. 5) have shown that for higher mass flow rates the Compton cooling leads to a steep decrease of the evaporation rate inwards. For our investigation this means that then the basic grid of $\log f$ curves (Fig. 1) would also have a more pronounced decrease towards smaller $r$. This is the region where we have not yet constructed consistent solutions. Having solved the problem of solutions for smaller $r$ it is meaningful to discuss the effect of Compton cooling on consistent coronal flow solutions.

\subsection{Spectral transitions in X-ray binaries}

The two examples for coronal flow corresponding to disk truncation at $\log r_{\text {tr }}$ equal to 8.5 and 9.1 (see Fig. 3) show the difference in the mass flows in disk and corona. For a mass flow of about $9 \times 10^{17} \mathrm{~g} / \mathrm{s}$ the thin disk becomes truncated at $\log r_{\mathrm{tr}}=9.1$. The hard spectrum is determined by this amount of mass flow in the corona, farther inward in the ADAF. If the mass flow in the thin disk is $2.4 \times 10^{18} \mathrm{~g} / \mathrm{s}$ the disk is truncated at $\log r_{\mathrm{tr}}=8.5$ (compare Fig. 3 for the amount of wind loss), the spectrum correspondingly changed. A factor of about 2.5 in the mass flow rate might occur during the increase or decrease to/from an outburst in an X-ray transient (even larger if wind loss is included). This leads to the change of the spectrum. For very high rates the disk is not truncated. For this case we can not yet present a solution.

\subsection{Concluding remarks}

The newly developed method for the construction of radially extended coronal flows allows a better description for the change from accretion via a thin disk to a disk free pure coronal flow (ADAF). We have presented results for disk truncation at various distances $r$ from a stellar black hole of $6 M_{\odot}$ applicable in X-ray binaries. The method also allows to study other regimes of solutions and might shed light on wind loss under those conditions. Particularly important will be to investigate whether the corona can partially condense into the thin disk again or keeps flowing at a significant rate. The solutions scale with Eddington accretion rate and Schwarzschild radius, are therefore also relevant for the accretion flow in AGN.

\section{References}

Deufel, B., \& Spruit, H. C. 2001, A\&A, 387, 918

Di Matteo, T., Fabian, A. C., Rees, M. J., et al. 1999, MNRAS, 305, 492

Di Matteo, T., Quataert, E., Allen, S. W., et al. 2000, MNRAS, 311, 507

Esin, A. A., McClintock, J. E, \& Narayan, R. 1997, ApJ, 489, 865

Esin, A. A., Narayan, R., Ciu, W., et al. 1998, ApJ, 505, 854

Gammie, C. F., Narayan, R., \& Blandford, R. 1999, ApJ, 516, 177

Liu, B. F., \& Meyer-Hofmeister, E. 2001, A\&A, 372, 386

Liu, B. F., Yuan, W., \& Meyer, F. 1999, ApJ, 527, L17

Liu, B. F., Mineshighe, S., Meyer, F., et al. 2002, ApJ, 575, 117

Meyer, F., \& Meyer-Hofmeister, E. 1994, A\&A, 288, 175

Meyer, F., \& Meyer-Hofmeister, E. 2001, A\&A, 380, 739

Meyer, F., \& Meyer-Hofmeister, E. 2002, A\&A, 392, L5

Meyer, F., Liu, B. F., \& Meyer-Hofmeister, E. 2000a, A\&A, 354, L67

Meyer, F., Liu, B. F., \& Meyer-Hofmeister, E. 2000b, A\&A, 361, 175

Meyer-Hofmeister, E., \& Meyer, F. 1999, A\&A, 348, 154

Meyer-Hofmeister, E., \& Meyer, F. 2001, A\&A, 380, 739

Narayan, R. 2002, in Lighthouses of the Universe, Conf. Proc., ed. M. Gilfanov, R. Sunyaev, \& E. Churazov, ESO Astrophys. Symp. (Springer), 405

Narayan, R., McClintock, J. E., \& Yi, I. 1996, ApJ, 457, 821

Narayan, R., Barret, D., \& McClintock, J. E. 1997, ApJ, 482, 448

Quataert, E., Di Matteo, T., Narayan, R., et al. 1999, ApJ, 525, L89

Rybicki, G. B., \& Lightman, A. P. 1979, Radiative Processes in Astrophysics (New York: Wiley)

Różańska, A., \& Czerny, B. 2000a, A\&A, 360, 1170

Różańska, A., \& Czerny, B. 2000b, MNRAS, 316, 473

Stepney, S. 1983, MNRAS, 202, 467 